\title{
Analysis of Precipitation and Dissolution in Overaged 7xxx Aluminium Alloys Using DSC
}

\author{
Xiaomei Li and M.J. Starink \\ Materials Research Group, School of Engineering Sciences, \\ University of Southampton, Southampton S017 1BJ, UK
}

Keywords: $\quad$ DSC, 7xxx alloys, precipitation, strengthening, alloy chemistry

\begin{abstract}
To improve the understanding of the relation between composition, precipitation and the balance of strength and electrical conductivity (as a measure of the stress-corrosion resistance), a number of $\mathrm{Al}-\mathrm{Zn}-\mathrm{Mg}-\mathrm{Cu}-\mathrm{Zr}$ aluminium alloy plates with different $\mathrm{Zn}, \mathrm{Mg}$ and $\mathrm{Cu}$ contents were produced and studied by Differential Scanning Calorimetry (DSC). It is shown that detailed analysis of the DSC data produces valuable information on the optimal $\mathrm{Mg}$, $\mathrm{Zn}$ and $\mathrm{Cu}$ contents.
\end{abstract}

\section{Introduction}

With the ever increasing demands put on high strength aluminium alloys for aerospace applications, improvement in alloy chemistry and thermo-mechanical treatments for optimisation of the critical properties strength, toughness and stress-corrosion cracking (SCC) resistance of 7xxx alloys is an area of continued interest. Differential Scanning Calorimetry (DSC) can provide a contribution to this [1] because it is sensitive to reactions of the main phases determining these properties, GP zones, $\eta^{\prime}, \eta, T, S$ and $\mathrm{Mg}_{2} \mathrm{Si}$ phase. In the present paper we will investigate to what extend DSC data can provide information concerning optimising alloy chemistry and heat treatment.

\section{Experimental}

Four Al-Zn-Mg-Cu-Zr plate alloys with different $\mathrm{Zn}, \mathrm{Mg}$ and $\mathrm{Cu}$ contents (broadly within in the range of 7075, 7010 and 7050 compositions) were studied. The alloys were produced at DERA (Farnborough, UK) under contract through British Aluminium Plate. The chemical compositions of the alloys are shown in Table 1 . All alloys were solution treated and water-quenched at DERA according to normal industrial practice.

For DSC testing, we cut samples from the plates and aged them for various times at a single temperature typical for a T7 temper. For all the experiments, a pure aluminium reference (99.9\%) with a mass and shape close to that of the sample was used. Baseline correction was performed employing experiments consisting of a single DSC run using pure Al samples. The heat flow was calibrated by measuring the heat of fusion of In and $\mathrm{Zn}$ as well as the heat capacity of pure Al. The temperature is calibrated by taking the deviation $\Delta \mathrm{T}$ from the reference temperature, compared with the measured melting points of In and Zn. The DSC samples were machined from the 7xxx plates to discs $5 \mathrm{~mm}$ in diameter and $1 \mathrm{~mm}$ in height with average mass of about $63 \mathrm{mg}$. DSC experiments were performed at heating rate $10^{\circ} \mathrm{C} / \mathrm{min}$. As standard procedure, each experiment contains 3 heating runs, with the latter two runs after cooling at 20 and $2^{\circ} \mathrm{C} / \mathrm{min}$ being used mainly for heat capacity corrections (for further details, see Ref. [2]).

For optical microscopy and image analysis, two alloys (B and D) were selected. For grain structure examination, polished samples were etched in $10 \% \mathrm{H}_{3} \mathrm{PO}_{4}$ in distilled water heated to $50^{\circ} \mathrm{C}$ for about one minute. 
Table 1 Chemical compositions of the alloys

$\begin{array}{lllllllll}\text { Alloy } & \mathrm{Zn} & \mathrm{Mg} & \mathrm{Cu} & \mathrm{Zr} & \mathrm{Ti} & \mathrm{Fe} & \mathrm{Si} & \mathrm{Zn}: \mathrm{Mg} \\ \mathrm{A} & 5.0 & 3.0 & 1.88 & 0.12 & 0.2 & 0.050 & 0.025 & 1.7 \\ \mathrm{~B} & 5.9 & 2.3 & 1.79 & 0.12 & 0.2 & 0.056 & 0.023 & 2.5 \\ \mathrm{C} & 6.7 & 2.0 & 1.88 & 0.12 & 0.2 & 0.074 & 0.024 & 3.3 \\ \text { D } & 6.1 & 2.3 & 2.58 & 0.12 & 0.2 & 0.071 & 0.020 & 2.6\end{array}$

Scanning Electron Microscopy (SEM) analysis of coarse intermetallic phases was performed for alloy D using a Jeol JSM-6400. Chemical compositions of various particles in the samples were measured using energy dispersive X-ray analysis (EDX).

\section{Results.}

Optical microscopy revealed that all alloys have largely similar grain structure: partially recrystallised with $60-80 \%$ recrystallised area fraction. Unrecrystallised grains show a pan-cake shape.

DSC experiments were performed on samples which were solution treated and aged for various ageing times. Fig. 1 shows a DSC curve of alloy B aged for 8 hours. Earlier work by other authors [3] evidences that for the present overaged alloys effect II corresponds to the dissolution of $\eta^{\prime}$, effect III corresponds to the formation of $\eta$ whilst effect IV is the dissolution of $\eta$. Since all alloys were in the overaged condition, formation (effect I) and dissolution of GP zones and most of the precipitation of $\eta^{\prime}$ have been completed prior to DSC experiments and thus these reactions are not observed. Two further effects, V and VI, are observed only in alloy D (Fig. 2). In view of the high $\mathrm{Cu}$ content of this alloy, these two effects are thought to be due to the formation and subsequent dissolution of S phase. Effect VIII is due to the melting of S phase [4] and the presence of this phase has been confirmed in SEM/EDX experiments on alloy D (The second type of intermetallic found was $\mathrm{Al}_{7} \mathrm{Cu}_{2} \mathrm{Fe}$. Melting of this phase is not observed in DSC experiments because the reaction is obscured by melting of the Al matrix phase). Effect VII has been observed for alloys with high Mg contents which contain T phase, and hence this peak is interpreted as being due to the melting of this Mg-rich phase.

Fig. 3 and Fig. 4 reveal that on increasing ageing times, effect III systematically decreases, and this is attributable to the formation of $\eta$. On increasing ageing times the total heat of $\eta^{\prime}$ dissolution (effect II) increases whilst the effect shifts to higher temperatures. This is due to a combination of precipitation of $\eta^{\prime}$ (which will increase effect II), growth of $\eta^{\prime}$ (which shifts effect II to higher temperatures) and formation of $\eta$ (which enhances effect II by reducing the overlap with effect III). It is further noted that variations in the effects that occur between 400 and $500^{\circ} \mathrm{C}$ do not depend on ageing time in a systematic manner. These variations are attributed to variations in the composition of the samples, which were taken from different positions within the plates.

The influence of varying Zn:Mg ratio on overaged 7xxx alloys is compared in Fig. 5 and Fig. 6. In these figures, $\mathrm{Zn:Mg}$ ratio increases in the sequence alloy A, alloy B, alloy C. The Cu content for three alloys is constant at $1.9 \mathrm{wt} \%$. It is observed that alloy A presents a sharp endothermic peak (onset temperature $490^{\circ} \mathrm{C}$ ) which corresponds to the melting of $\mathrm{S}$ phase. The presence of undissolved S phase at this temperature is due to the high $\mathrm{Mg}$ content of the alloy, which causes the alloy composition to be in the $(\alpha+S)$ phase field at the solution treatment temperature. In accordance with this interpretation, the heat effect of $\mathrm{S}$ melting decreases with increasing the Zn:Mg ratio. Fig. 5 and Fig. 6 further show that the overlapping $\eta^{\prime}$ dissolution effect (effect II) and $\eta$ formation effect (effect III) depend on Zn:Mg ratio. The alloys are all in overaged condition and $\eta^{\prime}$ will be largely completed for these alloys. Hence, differences in Fig. 5 and Fig. 6 are thought to be mostly due to enhanced rate of $\eta$ formation with increasing $\mathrm{Zn}: \mathrm{Mg}$ ratio. 

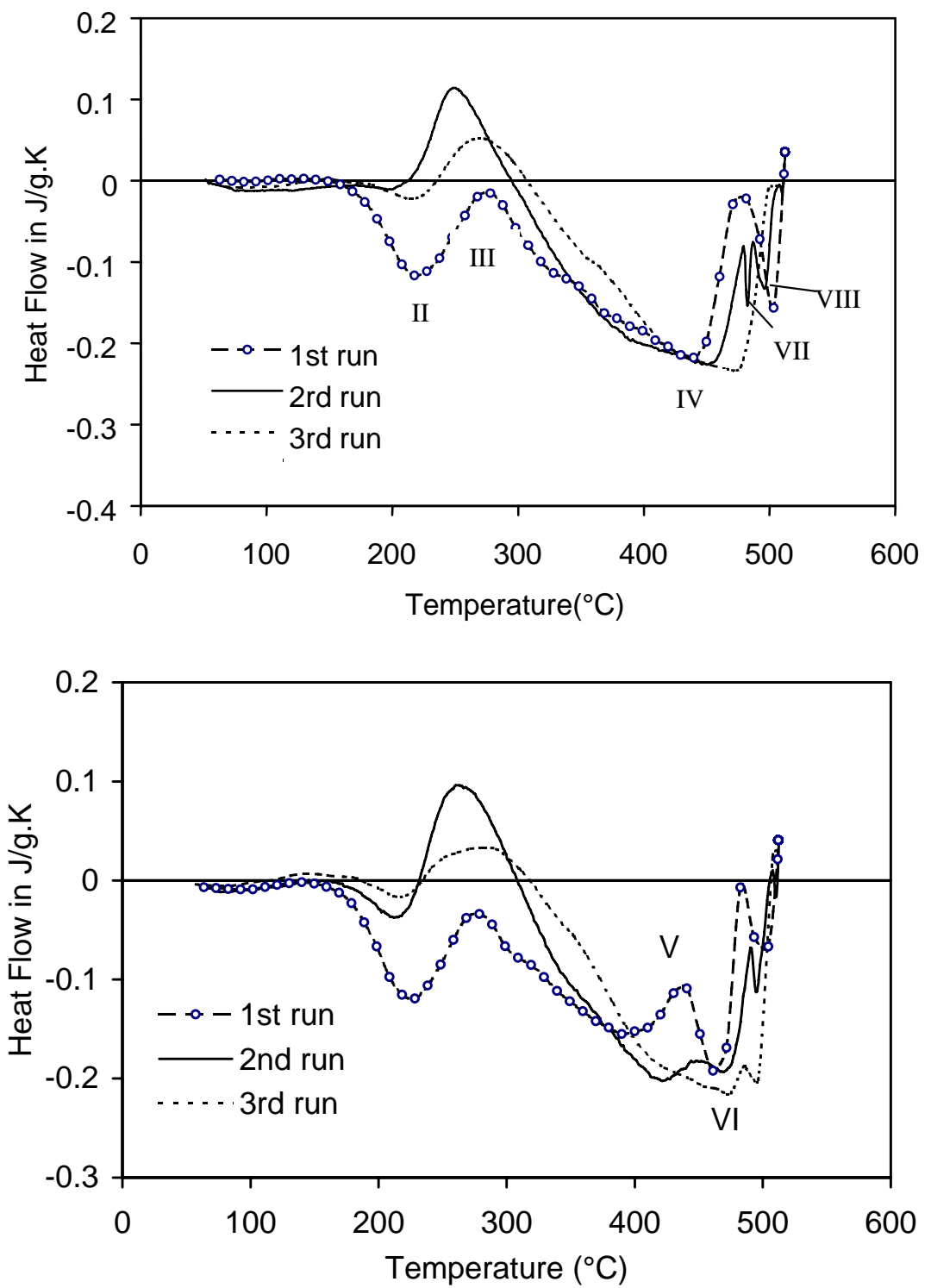

Fig. 1

DSC curves of runs 1 to 3 on alloy $B$ aged for $8 \mathrm{~h}$. Heating rate $10^{\circ} \mathrm{C} / \mathrm{min}$
Fig. 2

DSC curves of runs 1 to 3 on alloy $D$ aged for 8 h. Heating rate $10^{\circ} \mathrm{C} / \mathrm{min}$

The DSC curves for the overaged alloys with medium and low Mg content are compared in Fig. 5. This figure shows that the S phase melting peak (effect VIII) is especially strong in the high $\mathrm{Cu}$ alloy, alloy D. At this stage of the ageing, differences in the $\eta^{\prime}$ dissolution effect between the medium and low Mg alloys are limited.

\section{Discussion.}

In terms of the balance of the main properties of 7xxx alloys (strength, toughness and SCC resistance/conductivity) the present results indicate some interesting points. Firstly, it is clear that the high $\mathrm{Cu}$ and high $\mathrm{Mg}$ alloy contain significant amounts of coarse $\mathrm{S}$ phase which will degrade the toughness of the alloys. Hence these compositions are ruled out for most high strength aerospace applications. $\mathrm{Mg}$ and $\mathrm{Cu}$ incorporated in $\mathrm{S}$ phase will have very little effect on strength and conductivity of the alloy. Further, excess $\mathrm{Mg}$ is known to have a strong influence on conductivity [5] (high conductivity is related to susceptibility to SCC) thus indicating that low $\mathrm{Zn}: \mathrm{Mg}$ ratio is detrimental to this property. A further consideration is that to achieve high strength, high amounts $\eta^{\prime} / \eta$ should be able to precipitate and hence that high amounts of $\mathrm{Mg}$ and $\mathrm{Zn}$ should be dissolved in the Al-rich phase of the alloys. In DSC curves the amount of $\mathrm{Mg}$ and $\mathrm{Zn}$ that can be dissolved is reflected by a high endothermic heat of the dissolution of $\eta$ phase between about 300 and $490^{\circ} \mathrm{C}$. Since the end of the $\eta$ phase dissolution effect in alloy B is situated about $10^{\circ} \mathrm{C}$ beyond 
that of alloy C we may expect that alloy B will have a somewhat higher strength than alloy C. Thus the optimal Zn:Mg ratio should be situated approximately between those for alloy B and C (probably closer to $\mathrm{C}$ than to $\mathrm{B}$ ), with the higher $\mathrm{Zn:Mg}$ ratio improving the conductivity and the lower $\mathrm{Zn:Mg}$ ratio improving the strength.

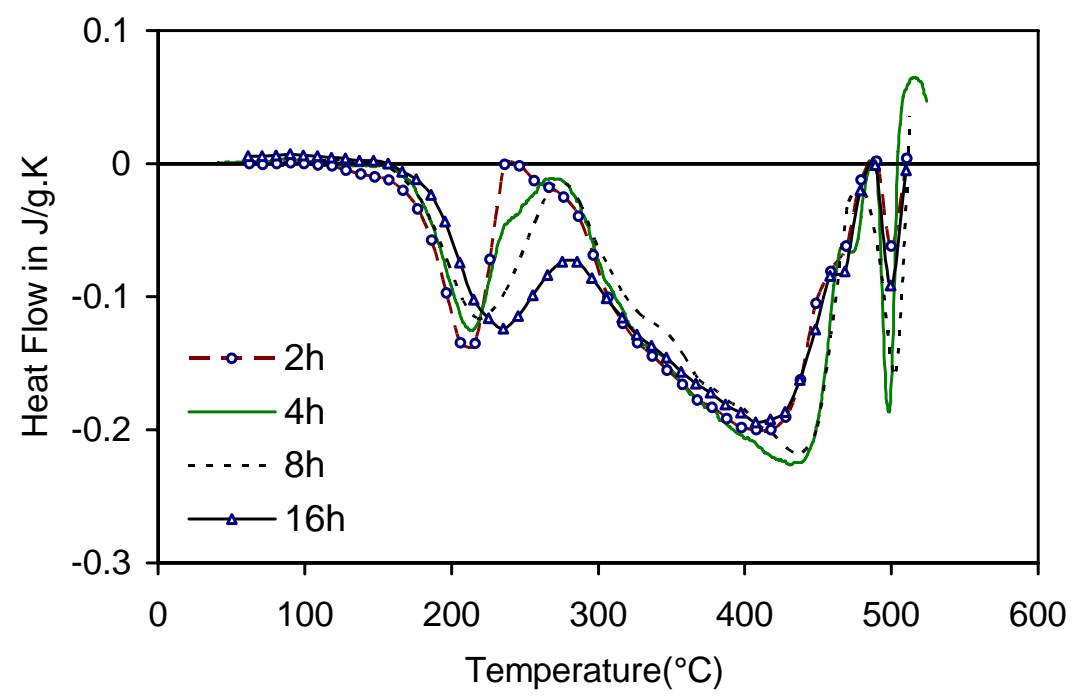

Fig. 3

DSC curves of alloy B aged for various times. Heating rate $10^{\circ} \mathrm{C} / \mathrm{min}$.

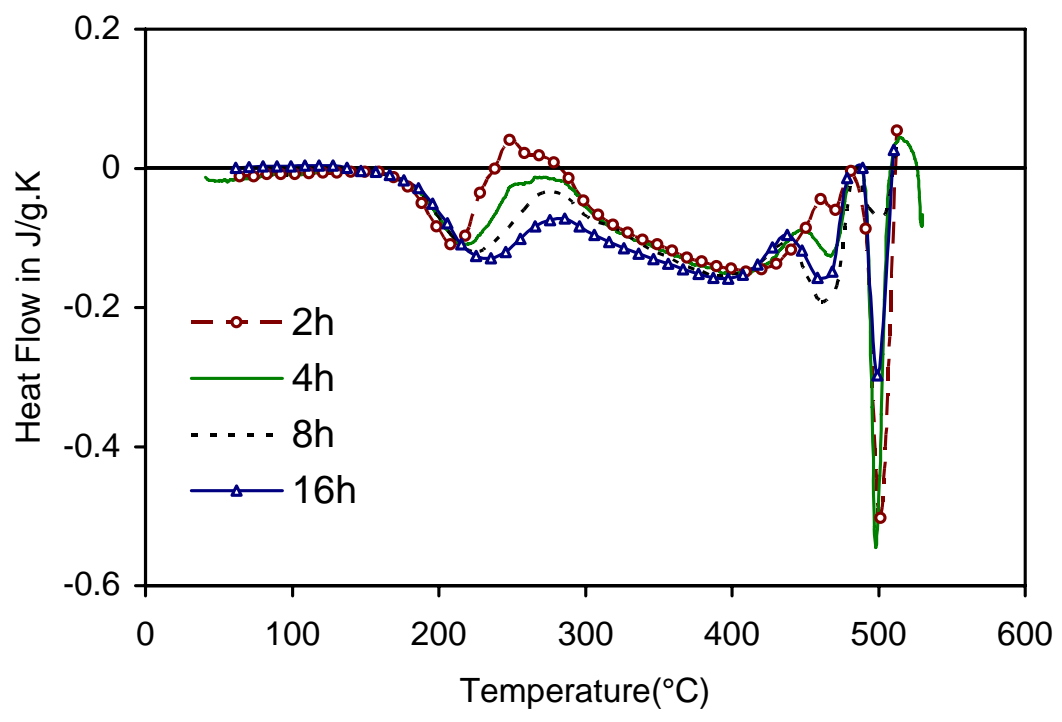

Fig. 4

DSC curves of alloy D aged for various times. Heating rate $10^{\circ} \mathrm{C} / \mathrm{min}$.

Exploring the optimisation of Mg and Zn contents further, the DSC curves indicate that for alloy B $\eta$ phase dissolution is completed at about $460^{\circ} \mathrm{C}$, i.e. before the solution treatment temperature or incipient melting temperatures are reached. Hence, there is some scope for increasing the $\mathrm{Mg}$ and $\mathrm{Zn}$ content of the alloy. The magnitude of this increase can be estimated using phase diagrams, however, in keeping with the aims of the present paper, we will here show that a reasonable estimate can also be obtained directly from the present DSC data. For this we approximate the $\eta$ phase dissolution effect by a triangle, as illustrated in Fig. 8. (Dissolution effects of equilibrium phases in many Al based alloys roughly approximate this shape, see e.g. Refs. 6,7). We can thus estimate:

$$
\frac{x_{Z n}^{g}(\text { optimised alloy })}{x_{Z n}^{g}(\text { alloy } B)}=\frac{\Delta Q(\text { optimised alloy })}{\Delta Q(\text { alloy } B)}=\frac{\left(T_{e}(\text { alloy } B)-T_{1}\right)^{2}}{\left(T_{s t}-T_{1}\right)^{2}}
$$


where $\mathrm{T}_{\mathrm{ST}}$ is the solutionising temperature and $x_{\mathrm{Zn}}^{g}$ is the gross $\mathrm{Zn}$ content of the alloy. Using $\mathrm{T}_{\mathrm{ST}}=475^{\circ} \mathrm{C}$ this estimate indicates that both the $\mathrm{Zn}$ and $\mathrm{Mg}$ content can be increased by about $12 \%$. However, we should additionally consider that $\mathrm{Mg}$ (and $\mathrm{Cu}$ ) content should remain below the $\mathrm{S}$ phase solvus, and hence $\mathrm{Cu}, \mathrm{Mg}$ and $\mathrm{Zn}$ composition should be limited. Thus, in order to optimise the balance of strength-toughness-SCC resistance alloy compositions around Al-7wt\%Zn-2.3wt\%Mg-1.7wt\%Cu should be considered. If processing can be carried out such that $\mathrm{T}$ phase is absent, solution treatment temperatures can be increased to just below the melting point of $\mathrm{S}$ phase. For $\mathrm{T}_{\mathrm{ST}}=485^{\circ} \mathrm{C}, \mathrm{S}$ phase solvus is no longer a limiting factor, and the optimised composition then becomes about Al-7.9wt\%Zn-2.5wt\%Mg-1.8wt\%Cu. (Phase diagrams indicate that the presence of $\mathrm{T}$ phase is induced by segregation of $\mathrm{Mg}$ and hence increasing $\mathrm{T}_{\mathrm{ST}}$ to $485^{\circ} \mathrm{C}$ is generally only possible for thinner plate.)

Obviously, the present interpretations based on DSC data alone need to be verified by comparison with data on mechanical properties of a wide range of 7xxx type alloys. This data is available with the sponsors of the current project, and this verification process will be the subject of further work.
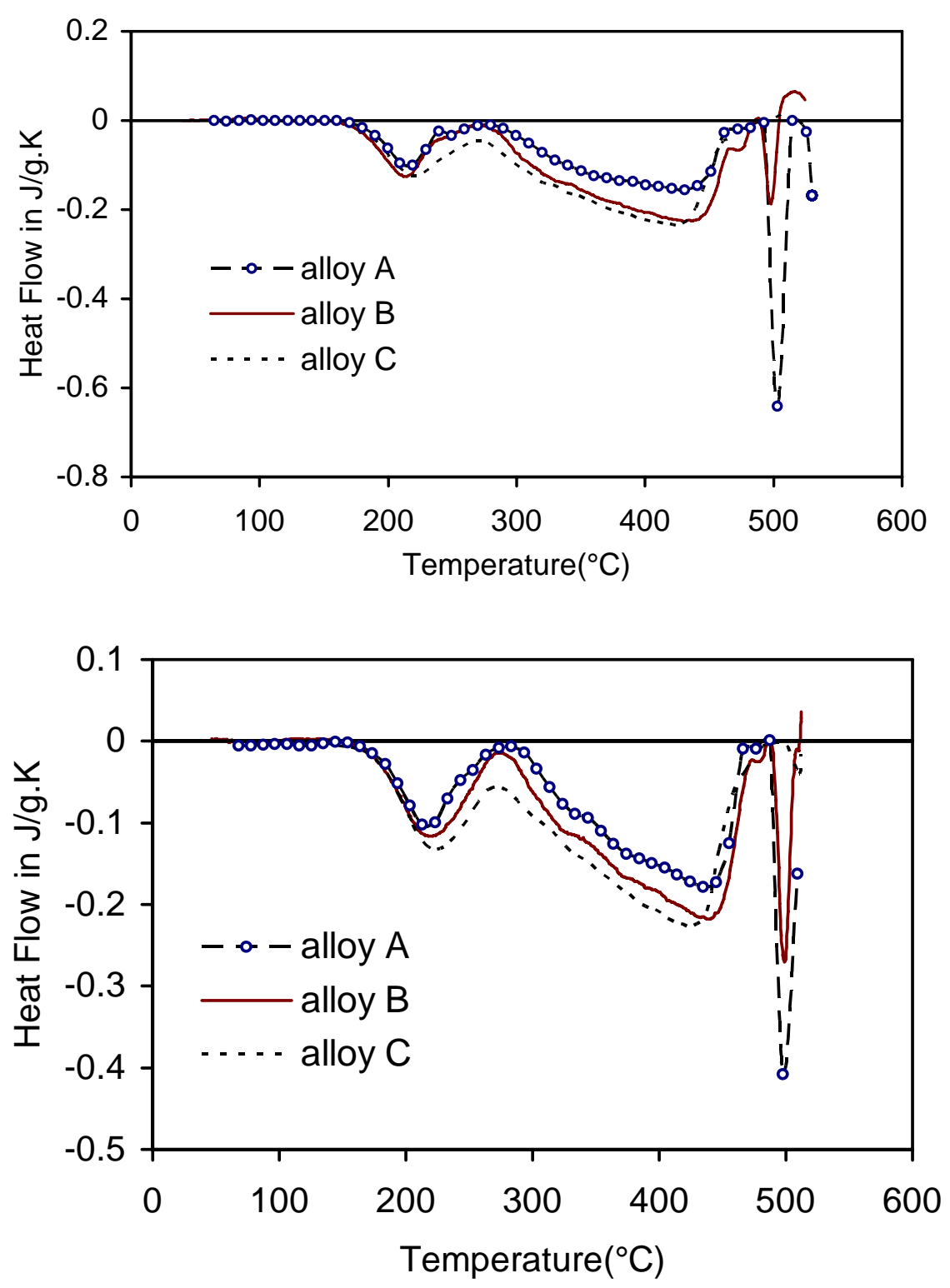

Fig. 5

DSC curves of alloy A, $B$ and $C$, aged for $4 \mathrm{~h}$. Heating rate $10^{\circ} \mathrm{C} / \mathrm{min}$.

Fig. 6

DSC curves of alloys A, $B$ and $C$, aged for $8 \mathrm{~h}$. Heating rate $10^{\circ} \mathrm{C} / \mathrm{min}$. 

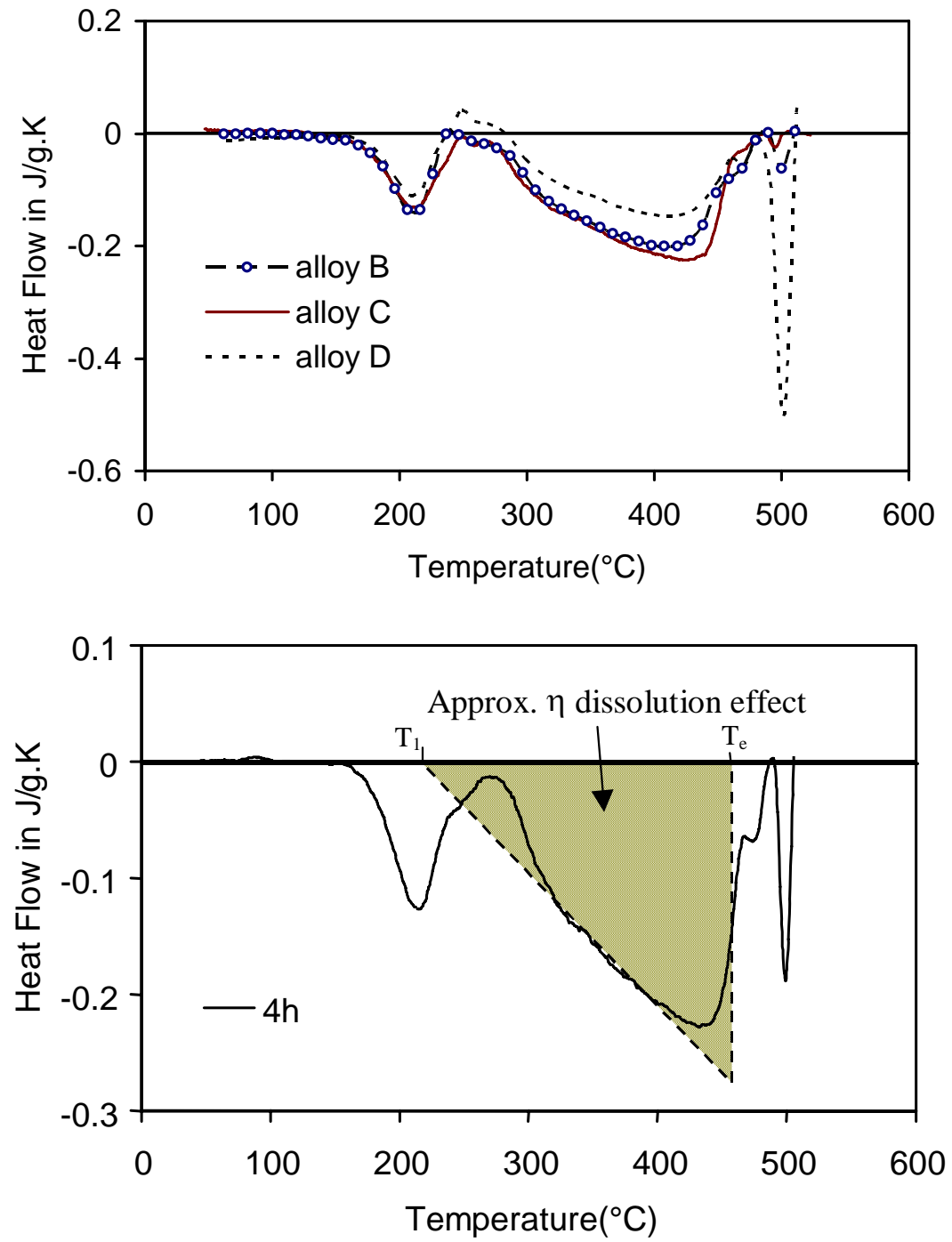

Fig. 7

DSC curves of alloys B, $\mathrm{C}$ and $\mathrm{D}$, aged for $2 \mathrm{~h}$. Heating rate $10^{\circ} \mathrm{C} / \mathrm{min}$.
Fig. 8

DSC curves of alloy B aged for $4 \mathrm{~h}$, together with an approximation of the heat of $\eta$ dissolution using a triangular heat effect.

\section{Conclusions}

DSC can be used to investigate the optimisation of the critical properties strength, toughness and stress-corrosion cracking resistance of 7xxx (Al-Zn-Cu-Mg) aluminium alloys by considering $\mathrm{S}$ and $\mathrm{T}$ phase content, incipient melting and amounts of $\eta$ phase. The present analysis indicates that optimised properties are obtained for $\mathrm{Zn:Mg}$ ratios around 3 and compositions around $\mathrm{Al}-7.3 \mathrm{wt} \%$ 2.4wt\%Mg-1.8wt\%Cu.

\section{Acknowledgements}

The financial support of British Aluminium Plate as part of the Luxfer group ATC programme is gratefully acknowledged. Technical input from Drs S. Waters and J. Newman (BAP) and P. Pitcher (DERA, Farnborough, UK) is gratefully acknowledged.

\section{References}

1 A.K. Gupta, A.K. Jena and D.J. Lloyd, Proc. ICAA-6, Toyohashi, Japan, July 5-10, 1998, p. 663

2 M.J. Starink and P.J. Gregson, Mater. Sci. Eng. A211 (1996) p. 54

3 J.K. Park and A.J. Ardell, Scr. Metall. 22 (1988) p. 1115

4 A.J. Morris, R.F. Robey, P.D. Couch and E. De los Rios, Mater. Sci. Forum 242 (1997) p. 181

5 O.P. Femminella, M.J. Starink, M. Brown, I. Sinclair, C.J. Harris and P.A.S. Reed, ISIJ Int. 39 (1999) p. 1027

6 M.J. Starink and A.-M. Zahra, Acta Mater. 46 (1998) p. 3381

7 M.J. Starink and P. van Mourik, Mater. Sci. Eng. A156 (1992) p. 183 